\title{
Ultrastructure of Obstructive Tissue in Malfunctioning Ventricular Catheters Without Infection
}

\author{
Yoshihiko TAKAHASHI, Akira OHKURA, Masaru HiRohata, Takashi TOKUTOMI, \\ and Minoru SHIGEMORI
}

Department of Neurosurgery, Kurume University School of Medicine, Kurume, Fukuoka

\begin{abstract}
The obstructive tissue in eight malfunctioning ventricular catheters without infection was studied using scanning and transmission electron microscopy. Shunt obstruction was due to debris from ventricular structures such as the choroid plexus and ependymal tissue. There was a preponderance of collagen fibers, and many fibroblasts were present within these tissues. The cytoplasm of the fibroblasts contained extended endoplasmic reticulum. The tissues filled the lumen of the catheters in radially arranged layers. Peeling of the silicone was seen on the surface of the ventricular catheters but was not present in normal silicone catheters. Finger-like microvilli were observed on the free surface of the tissue. Many vessels were seen in the transverse section of the tissue. Activated fibroblasts and vascularization may be important in tissue growth in ventricular catheters.
\end{abstract}

Key words: ventriculoperitoneal shunt, shunt malfunction, ventricular catheter, choroid plexus, silicone, hypersensivity reaction

\section{Introduction}

Ventriculoperitoneal shunting is commonly used for the treatment of hydrocephalus. Recently, the use of silicone-coated shunt catheters has reduced complication rates, but in a combined series of 1700 patients, the shunt failure rate was $40 \%$ in the first year. However, the incidence of shunt malfunction after 1 year was significantly lower, at only $5 \% .{ }^{1]}$ The sites of shunt malfunction were divided into four groups: proximal obstruction, valve obstruction, distal obstruction, and undefined obstruction. ${ }^{11)}$ Proximal obstruction was the most common site of failure. Obstruction of the ventricular shunt catheter is the most significant problem associated with shunts for hydrocephalus, but a number of factors usually combine to obstruct the shunt.

Previously, histological studies of the malfunctioning shunt system have been made. ${ }^{2,5-9,12)}$ However, few ultrastructural studies have investigated delayed malfunctioning ventricular shunt catheters without infection using scanning electron microscopy (SEM) and transmission electron microscopy (TEM). Observations of the surface of oc-

Received December 22, 1997; Accepted April 8, 1998 cluded ventricular catheters by SEM showed that the ventricular catheter was occluded by ependymal cells, choroidal plexus, clots, and brain tissue, ${ }^{21}$ but the inner surface and transverse section of the catheters, and the tissue were not characterized.

This study investigated obstructed ventricular shunt catheters using SEM and TEM to clarify the three-dimensional features of this complication.

\section{Clinical Materials and Methods}

Forty-five shunt revisions were performed in 38 patients in our neurosurgical clinic from 1994 to 1995. Eight patients had malfunctioning ventriculoperitoneal shunts due to obstructed ventricular shunt catheters without infection. These five female patients and three male patients had a mean age of 19.2 years (range 9-26 years). The duration of implantation ranged from 2 years to 10 years.

The catheters were gently washed in saline, and placed in $2 \%$ glutaraldehyde for 12 hours. The catheters were sectioned along the plane of the long axis, placed on SEM stage posts, and dried with carbon dioxide in a critical-point apparatus. The specimens were subsequently sputter-coated with gold, and observed by SEM (HITACHI S-800; Hitachi, Ibaraki). 
Specimens were obtained from the inner surfaces of the catheters, placed in $2 \%$ glutaraldehyde, and then postfixed in buffered $2 \%$ osmium tetroxide for 2 hours. The specimens were then dehydrated in a graded alcohol series, and embedded in Epon 812. Ultrathin sections were stained with uranyl acetate and lead citrate, then examined by TEM (HITACHI H-7000; Hitachi). New catheters of the same type were examined as controls.

\section{Results}

\section{TEM observations}

Cells with many microvilli were clustered at the surface of the tissues in the catheters. Cilia were also observed in some places. The nuclei of these cells were flat and heterochromatin was seen at the margin of the nucleus. Cytoplasm combined well-developed glial filaments and mitochondria (Fig. 1 upper, middle). Fenestrated capillaries could be identified. These findings suggested that these cells originated from the choroidal plexus. A preponderance of collagen fibers and many fibroblasts were present in the core of the tissue obstructing the catheters. The fibroblasts had extended endoplasmic reticulum with distended cisternae (Fig. 1 lower). The tissue obstructing the catheter was composed of reactive glial tissue, mainly identified as choroidal ependyma, and fibroblasts which actively synthesized collagen fibers.

\section{SEM observations}

All catheters were obstructed by tissue which had filled the lumen in radially arranged layers. The tissue was present in the lumen adjacent to the inlet fenestration of the catheters (Fig. 2 upper, middle]. Finger-like microvilli were observed on the free surface of the tissue (Fig. 2 lower). Peeling of the silicone was seen on the inner surface of the ventricular catheters (Fig. 3 upper). Many small vessels were observed in the transverse section of the tissue (Fig. 3 lower). In contrast, the control catheter had a smooth inner surface (Fig. 4).

\section{Discussion}

\section{Obstructive tissue}

In our study, the obstructive tissue consisted of ependyma, fibroblasts, and collagen fibers as well as choroid plexus. Previous investigation of the ultrastructure of tissues occluding ventricular catheters showed that choroid plexus was frequently found in catheter tips placed behind the foramen of Monro, but ependymal tissue predominated in catheters placed in front of the foramen of Monro. ${ }^{2)}$ Such ob-

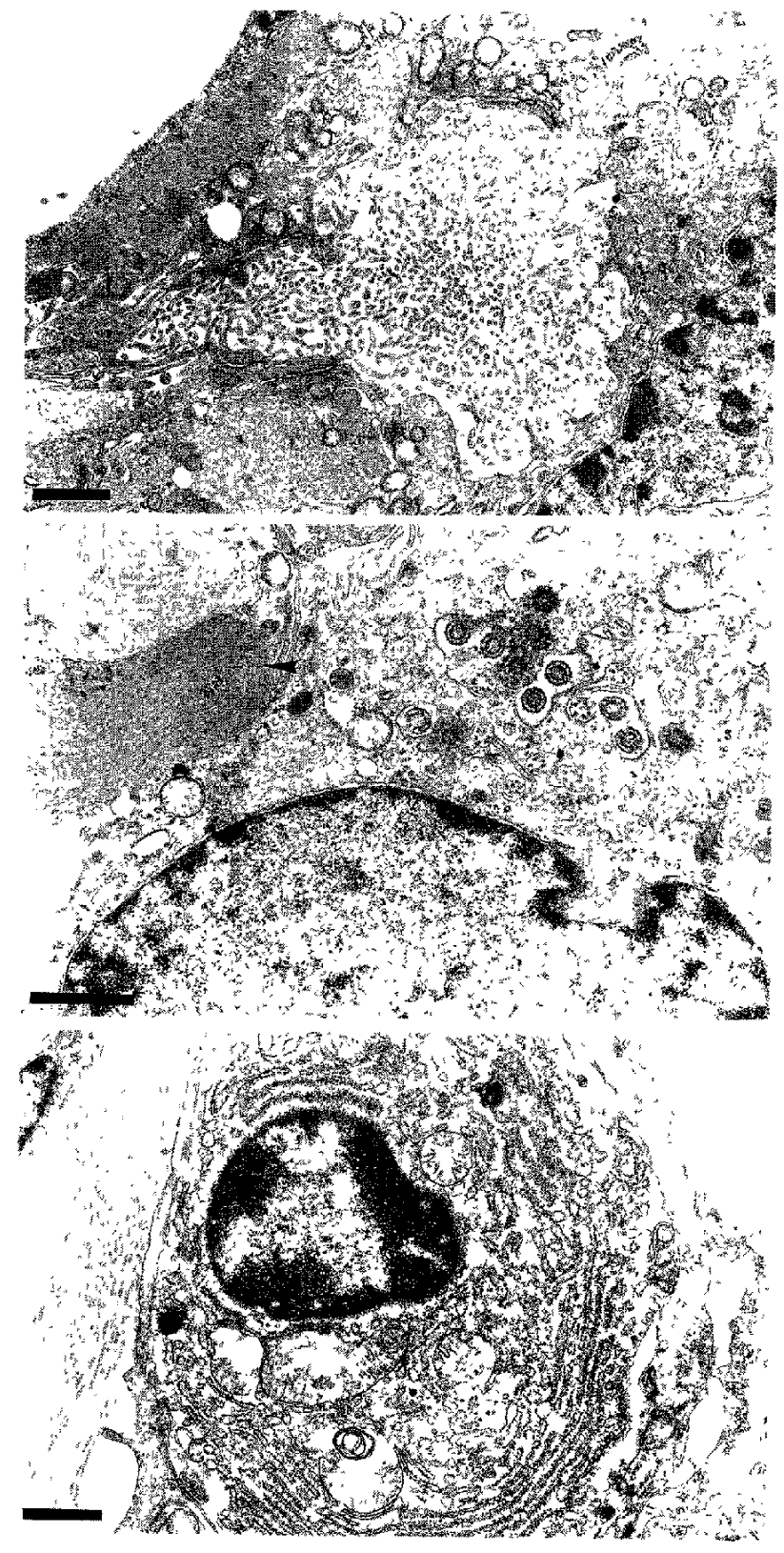

Fig. 1 Trasmission electron micrographs. upper: Many microvilli are observed at the surface of the tissues. Bar $=1 \mu \mathrm{m}$. middle: The nucleus is flat and heterochromatin is observed at the margin of the nucleus. Cytoplasms have well-developed glial filaments (arrowhead) and mitochondria. Cilia are also observed. Bar $=1 \mu \mathrm{m}$. lower: Fibroblasts have extended endoplasmic reticulum with distended cisternae. Bar $=1 \mu \mathrm{m}$.

servations suggest that the nature of the obstructive agent is related to the position of the catheter. Study of the reaction of the periventricle tissue of the later- 

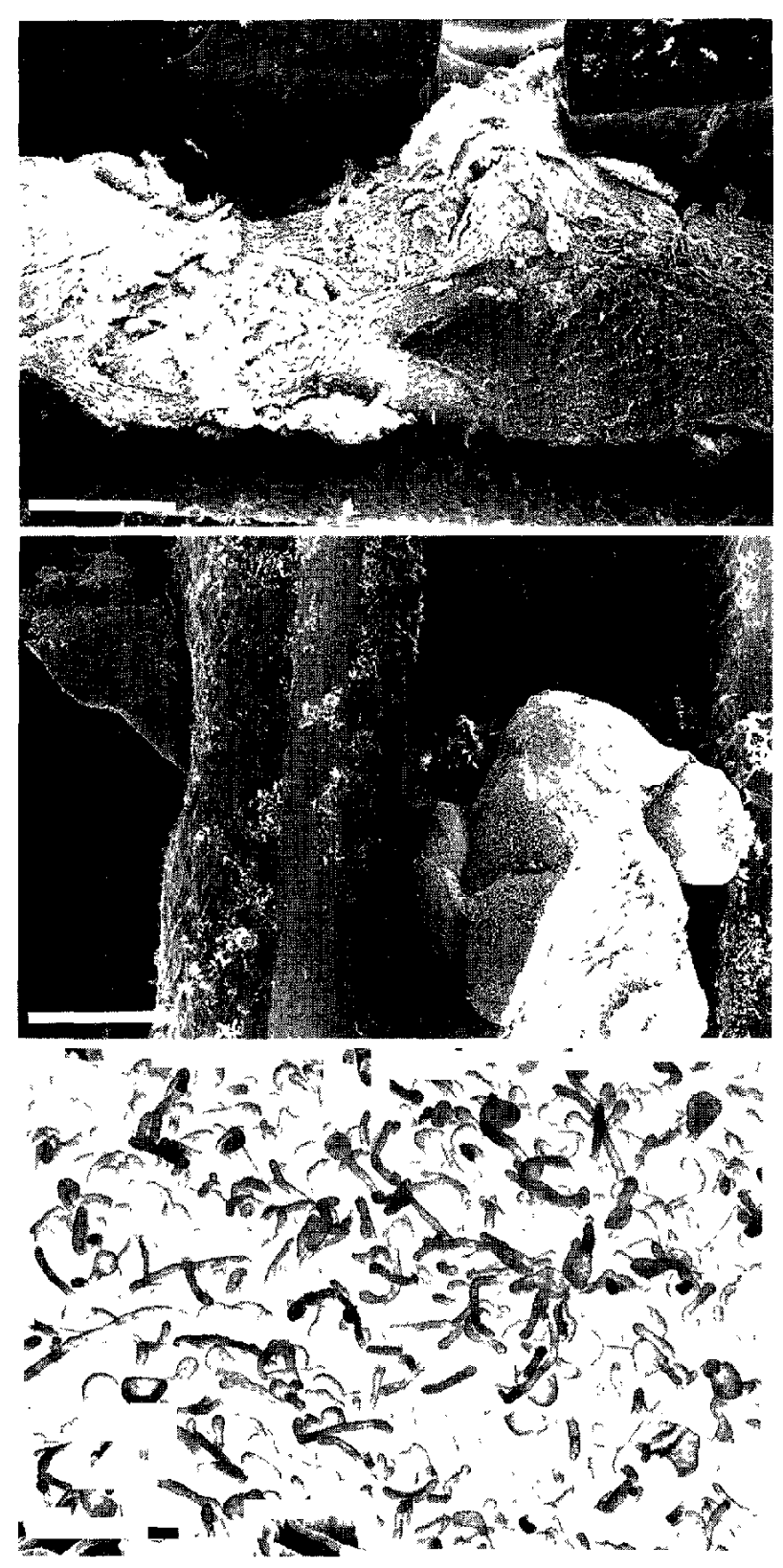

Fig. 2 Scanning electron micrographs. upper, middle: The catheters are obstructed by the tissue which has filled the lumen of the catheters in radial layers. The tissue is connected to the outer tissue through the fenestrations. (upper, Case 1; middle, Case 2) Bar $=\mathbf{5 0 0} \mu \mathrm{m}$. lower: Finger-like microvilli are observed on the free surface of the tissue. Bar $=5 \mu \mathrm{m}$.

al ventricle to silicone shunt tubes in the rabbit showed that 3 weeks post-implantation, a ventricular wall outgrowth was partially covered by ependyma. ${ }^{3)}$ In our patients, the ventricles were slit-like, and the catheter was in close contact with the

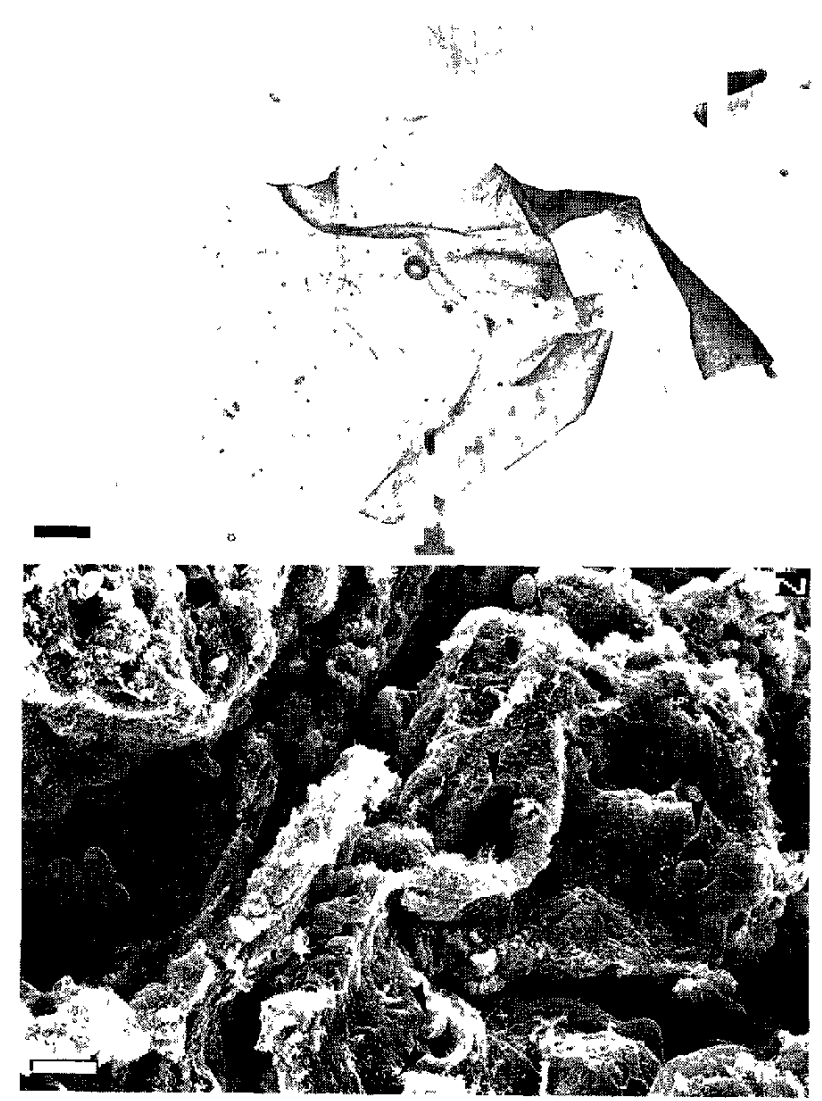

Fig. 3 Scanning electron micrographs. upper: Peeling of the silicone is seen on the inner surface of the ventricular catheters. Bar $=10 \mu \mathrm{m}$. Iower: Small vessels are observed in the transverse sections of the tissue (arrowheads). Bar $=10 \mu \mathrm{m}$.
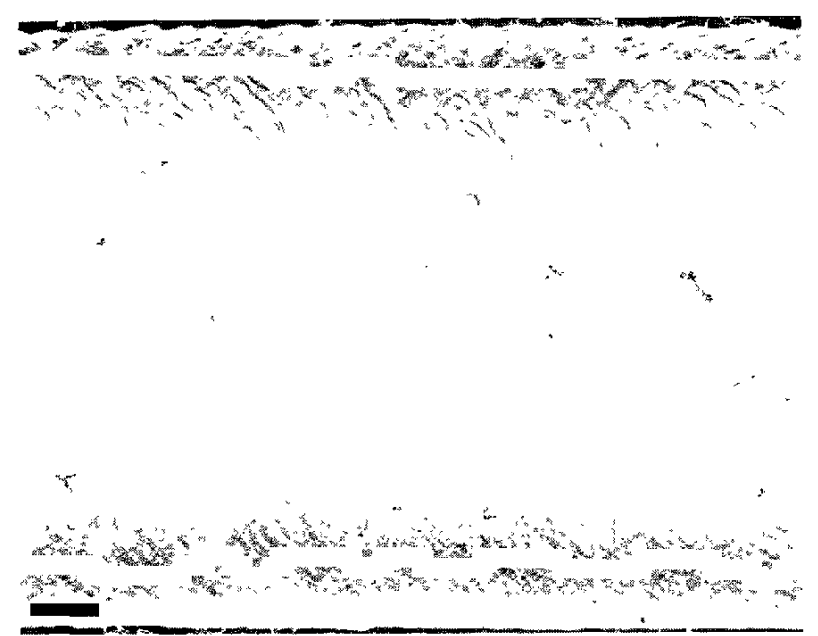

Fig. 4 Scanning electron micrograph. The inner surface of a normal ventricular catheter is smooth. $\quad$ Bar $=100 \mu \mathrm{m}$. 
choroid plexus. The risk of shunt failure was significantly higher in the case of slit ventricles. ${ }^{4,7,13}$ The location of the catheter tip is the other important factor in proximal shunt obstruction. ${ }^{1]}$

Normal choroidal plexus and ependyma generally contains few glial filaments. Our study found that the glial cells which obstructed the shunt catheters possessed many glial filaments in the cytoplasm, and were different from normal ependyma and normal choroidal plexus. Such morphological changes in the obstructing tissue may be the result of an interaction between the silicone ventricular tube and the ependyma. There are three types of cells in the epithelial layer of the human choroid plexus. ${ }^{101}$ Type 1 cells are cuboidal or columnar and lined the villi, a round nucleus with diffuse chromatin and a clear nucleus occupies the central area of the cell, and numerous club-shaped microvilli occur on the free surface. Type 2 cells are flat cells with many mitochondria filling the cytoplasm, many finger-like microvilli, and a thick basal lamina. Type 3 cells are flat with round or oval nuclei, and diffuse chromatin throughout the nucleus, with less prominent mitochondria, Golgi apparatus, and rough endoplasmic reticulum than in type 1 cells. These three types of cells have tight junctions and basal infolding. Most of the ependyma in the obstructed shunt catheters was similar to type 2 cells, but basal infolding and tight junctions were rarely seen. The type 2 cells lined connective tissue adjacent to neural tissue, and the connective tissue was subsequently inserted into the ventricular catheter after the choroid plexus.

\section{Growth factors of the obstructive tissue}

Hypersensitive reaction to silicone material may cause shunt obstruction, but other factors should be considered. This study found many small vessels and activated fibroblasts in the tissue which obstructed the ventricular catheter. These small vessels were characterized by fenestrations, suggesting that the vessels originated from the choroid plexus. The choroid plexus was potentially carried into the ventricular catheters by the small vessels and subsequently grew within the shunt tube. Fibroblasts may also be important in occlusion of the shunt catheter. The cytoplasm of the fibroblasts included extensive endoplasmic reticulum, so these fibroblasts may synthesize the collagen fibers, which also contributed to the obstructive tissues in the catheters.

\section{Peeling of silicone}

This study observed peeling of the surface of the silicone catheter. This phenomenon apparently resulted from the hypersensitive reaction between the silicone and the cerebrospinal fluid. Peeling of the silicone, proteinaceous debris, and fibrin nets were also found in the shunt catheter previously, ${ }^{9)}$ suggesting that this was caused by a biological reaction. Silicone materials are inert in the body, but delayed hypersensitive reactions have been reported. ${ }^{14 j}$ The reactive formation may progress slowly, and is related to a subacute eosinophilic reaction to a sterile shunt system. The incidence of cerebrospinal fluid eosinophilia is $6.4-34 \%$ in children with ventriculoperitoneal shunts, and may be related to hypersensitive reactions. ${ }^{15]}$ Giant cells with multiple foot processes may be adherent to the silicone catheter, suggesting that a delayed immune hypersensivity response like that observed with breast implants might help explain some shunt failures. ${ }^{5}$ Since the duration of the shunt malfunction is variable, the hypersensitive reaction may be different between patients. Therefore, a completely inert material may be required for shunt systems in the future.

\section{References}

1) Bierbrauer KS, Stross BB, McLone DG: A prospective, randomized study of shunt function and infections as a function of shunt placement. Pediar Neurosurg 16: 287-291, 1990

2) Collins P, Hockeley AD, Woollam DHM: Surface ultrastructure of tissues occluding ventricular catheters. J Neurosurg 48: 609-613, 1978

3) Del Bigio MR, Bruni JE: Reaction of rabbit lateral periventricular tissue to shunt tubing implants. J Neurosurg 64: 932-940, 1986

4) George R, Leiblock L, Epstein M: Long term analysis of cerebrospinal fluid shunts infections. J Neurosurg 51; 804-811, 1979

5) Gower DJ, Lewis JC, Kelly DL: Sterile shunt malfunction. A scanning electron microscopic perspective. I Neurosurg 61: 1079-1084, 1984

6) Griebel RW, Hoffmann HJ, Becker LE: Calcium deposits on CSF shunts. Childs Nerv Syst 3: 180-182, 1987

7) Griebel RW, Khan M, Tan L: CSF shunt complications: An analysis of contributory factors. Childs Nerv Syst 1: 77-80, 1985

8) Guevara JA, La Torre J, Denoya C, Zuccaro G: Microscopic studies in shunts for hydrocephalus. Childs Brain 8: 284-293, 1981

9) Koga H, Mukawa J, Nakata M: Analysis of retained ventricular shunt catheters. Neurol Med Chir (Tokyo) 32: 824-828, 1992

10) Matsushima T: Choroid plexus papillomas and human choroid plexus: A light and electron microscopic study. J Neurosurg 59: 1054-1062, 1983

11) Sainte-Rose C: Shunt obstruction: A preventable complication? Pediatr Neurosurg 19: 156-164, 1993

12] Sekhar LN, Moossy J, Guthkelch N: Malfunctioning ventriculoperitoneal shunts: Clinical and pathologi- 
cal features. J Neurosurg 56: 411-416, 1982

13) Serlo W, Fernell E, Heikkinen E; Functions and complications of shunts in different etiologies of childhood hydrocephalus. Childs Nerv Syst 6: 92-94, 1990

14) Snow RB, Kossovsky N: Hypersensitivity reaction associated with sterile ventricular peritoneal shunt malfunction. Surg Neurol 31: 209-214, 1989

15) Taynelis VC, Powell RG, Koss W, Schochet SS, Kaufman $\mathrm{H}$ : Cerebrospinal fluid eosinophilia and sterile shunt malfunction. Neurosurgery 23: 645-649, 1988

Address reprint requests to: Y. Takahashi, M.D., Department of Neurosurgery, Kurume University School of Medicine, 67 Asahi-machi, Kurume, Fukuoka 8300011, Japan.

\section{Commentary}

Proximal obstruction in ventriculoperitoneal shunts remains the most common cause of failure. The authors present an interesting study in which the obstructive material was studied using scanning and transmission electron microscopy in eight uninfected ventricular catheters.

This study leads to several conclusions:

1) Confirmation with electron microscopy of the correlation between the site of implantation and the obstructive tissue: Choroid plexus for ventricular catheters implanted into the ventricular body and ependymal tissue for frontal implantation.

2) Presence of obstructive tissue organized in a number of levels related with the length of evolution.

3) This material, besides the ependymal and choroid tissue, is represented by reactive tissue with glial cells, collagen, and fibroblasts. The cytoplasm of fibroblasts includes extensive endoplasmic reticulum, suggesting an important secretory activity.

4) The external layer of the ventricular catheter is not preserved, but shows peeling of the silicone surface, suggesting a biological reaction.

5) The reactive formation may progress during the evolution.

This article is interesting since it helps to explain some late complications in shunt procedures, independent of the technique of implantation, and may be helpful in the prevention of such obstruction with the use for the future of completely inert material.

Maurice CHoux, M.D.

Service de Neurochirurgie Infantile

Hôpitaux de Marseille Marseille, France

The authors have presented ultrastructural observa- tions of delayed malfunction in ventricular shunt catheters without infection. On the basis of SEM and TEM findings of the obstructive tissue found in eight malfunctioning ventricular catheters, they stressed the role of biological reactions of the host to the shunt tube. They consider that peeling of the surface of the silicone catheter was the result of a hypersensitive reaction between the silicone and the CSF. More valid evidence, such as the focal infiltration of eosinophils and/or lymphocytes, should have been presented in order to attach importance to host hypersensitivity as a causative factor of shunt obstruction. Immunohistochemical staining for markers such as GFAP andior vimentin needs to be done. In general, the primary factors associated with shunt obstruction are considered to be abnormal CSF components, such as protein at high concentration, and trouble with the shunt system. However, no details of these factors were mentioned. The etiology of hydrocephalus, which seems to be closely related to the incidence of shunt malfunction, should have been described. However, this work is an important addition to our knowledge of the morphological phenomena that play a major role in the delayed malfunction of ventricular shunt catheters in the absence of infection.

Kouzo Moritake, M.D. Department of Neurosurgery Shimane Medical University Shimane, Japan

The authors have used scanning and transmission electron microscopy to study the cause of shunt obstruction in eight ventricular catheters. They found that shunt obstruction was caused by cellular debris from choroid plexus and ependymal tissues. In addition, collagen fibers from fibroblasts were abundant, as were microvessels within the organized debris of the ventricular catheters. They describe a process of "peeling" of the ventricular catheters not previously reported.

This is an outstanding contribution to the literature on the causes of shunt failure. The images provided are excellent, and clearly demonstrate that standard ventricular catheters are not made of inert substances. The brain has a tremendous capacity to react to these catheters. The information from this study speaks to the importance of the ongoing randomized shunt insertion trial in North America in which it will be determined if shunt placement by endoscopic techniques away from the choroid plexus per se has a role to play in preventing shunt obstruction. I congratulate the authors on the results of this fine paper.

James T. RUTKA, M.D.

Division of Neurosurgery The Hospital for Sick Children

Neurol Med Chir (Tokyo) 38, July, 1998 
and Brain Tumor Research Laboratory University of Toronto

Ontario, Canada

The authors studied the ultrastructure of tissue in obstructed ventricular catheters and found that activated fibroblasts and angiogenesis are possibly the major cause for tissue growth. As the authors mentioned in this article, shunt malfunctions due to the obstruction of ventricular catheters are still annoying complications for neurosurgeons. Although the authors described that the choroid plexus biologically "grew" into the catheter, other mechanisms such as simple suction by the negative pressure of the catheter may exist. Nevertheless, the finding that the obstructed tissue was vascularized by vessels originating from the choroid plexus is important from the aspect of immunological reaction. Those vessels originating from the choroid plexus are supposed not to possess the blood-brain barrier and thus the antibodies in the serum may more easily recognize the shunt materials than in the case when the catheter was in the right place surrounded only by the tissue with blood-brain barrier. The correlation of vascularized tissue with the incidence of the hyperreaction or the peeling of catheter is thought to be the subject for further study.

Norihiko TAMAKI, M.D.

Takeshi KONDOH, M.D. Department of Neurosurgery Kobe University School of Medicine Kobe, Japan 